\title{
RECUPERAÇÃO DE ATRIBUTOS SÍSMICOS UTILIZANDO A MIGRAÇÃO PARA AFASTAMENTO NULO
}

\author{
Angela C. R.Vasquez, ${ }^{1}$ Adelson S. Oliveira, ${ }^{2}$ Martin Tygel ${ }^{3}$ e Lúcio T. Santos ${ }^{4}$ \\ Recebido em 18 fev., 2001 / Aceito em 7 ago., 2003 \\ Received Feb. 18, 2002 / Accepted Aug. 7, 2003
}

\section{ABSTRACT}

A conventional processing, without a reliable adjustment in order to preserve the seismic amplitudes, could damage the mapping of the petrophysical properties, jeopardizing the correlation between the seismic data and the well profile. A manner to estimate correctly the amplitudes and, therefore, the reflection coefficients is to perform a pre-stack migration in true amplitude, where an amplitude distortion caused by the geometrical spreading throughout the ray path is compensated by the migration calculation. Nevertheless, this process has an expensive cost as well as is dependent from the velocity model.

A routine less expensive than the other one and also more stable taking into account the velocity model, is to transform the seismic section obtained from the acquisition in common offsets in simulated section in zero offset with true amplitudes. This transformation is called true amplitude Zero Offset migration (TA MZO). In a media with constant velocity, the stack curve for the MZO and the weight function are reduced in analytic formulas, mitigating the computational effort. This work has two main objects: the first is to verify the TA MZO algorithm efficiency for a constant velocity in a synthetic model to a complex geology based on a Neo-Albian turbidity reservoir, where the assumption of constant velocity is not respected. The second one is to perform quantitative studies as results of the technique described above. Likewise, the study tries to analyze how useful is the methodology to compute the graphics for AVO and AVA analyses, helping the reservoir characterization.

Keywords: spreading; seismic; migration; coefficient.

\section{RESUMO}

0 processamento convencional, sem um tratamento adequado de preservação de amplitude, mascara ou até inviabiliza o mapeamento de propriedades petrofísicas, prejudicando a correlação entre o dado sísmico e a informação de perfil. Uma forma de estimar corretamente as amplitudes e, por conseqüência, os coeficientes de reflexão nas interfaces sísmicas, é efetuar uma migração pré-empilhamento em verdadeira amplitude, onde a distorção das amplitudes devido ao espalhamento geométrico ao longo do raio de reflexão é compensado pela operação de migração. Entretanto, esse processo tem um custo elevado, sendo também dependente do modelo de velocidades utilizado.

Uma rotina menos dispendiosa e mais estável em relação a erros no modelo de velocidade, consiste na transformação da seção sísmica obtida com a geometria de aquisição no afastamento comum em seções simuladas de afastamento nulo em amplitude verdadeira. A operação de transformação de uma seção sísmica de afastamento constante para a sua correspondente em afastamento nulo recebe o nome de migração para afastamento nulo em verdadeira amplitude (TA MZO). No caso de um meio com velocidade constante, a curva de empilhamento do MZO e a função peso se reduzem a fórmulas analíicas, minimizando 0 esforço computacional.

0 trabalho em questão tem dois objetivos principais: o primeiro é verificar a eficácia do algoritmo de TA MZO para velocidade constante em um modelo sintético com geologia complexa baseado em um reservatório turbidíitico brasileiro de idade Neo-Albiana, onde a hipótese de velocidade constante é desrespeitada. 0 segundo objetivo é a realização de estudos quantitativos referentes à obtenção de amplitudes resultantes da aplicação da rotina descrita acima, bem como avaliar sua utilização para a construçãa de gráficos de amplitude com 0 afastamento (AVO) e com o ângulo (AVA) em interfaces relevantes para a caracterização de reservatórios.

\footnotetext{
PETROBRAS Petróleo Brasileiro AS - Av. Chile 65, sala 1302 - Rio de Janeiro - RJ - CEP: 20.031-912 - Telefone: 21 2534-8013 - Email: avasquez@petrobras.com.br

PETROBRAS Petróleo Brasileiro AS - Av. Chile 65, sala 1402 - Rio de Janeiro - RJ - CEP: 20.031-912 - Telefone: 2125341135 - Email: adelson_so@petrobras.com.br

3 UNICAMP - IMECC - Instituto de Matemática, Estatística e Computação Científica - Praça Sergio Buarque de Holanda, 651 - Cidade Universitária - Barão Geraldo - Caixa Postal: 6065 - Campinas - São Paulo - 13083-859 - Email: tygel@unicamp.br

4 UNICAMP - IMECC - Departamento de Matemática Aplicada - Caixa Postal 6065 - Campinas - SP - CEP: 13081-970 - Telefone: (19) 3788-5975 - Email: lucio@ime.unicamp.br
} 
Palavras-chave: afastamento; sísmica; migracão; coeficiente.

\section{INTRODUÇÃO}

0 processamento convencional, sem um tratamento adequado de preservação de amplitude, mascara ou até inviabiliza o mapeamento de propriedades petrofísicas, prejudicando a correlaçãa entre 0 dado sísmico e a informação de perfil. Uma forma de estimar corretamente as amplitudes e, por consequiência, os coeficientes de reflexão nas interfaces sísmicas, é efetuar uma migração pré-empilhamento em verdadeira amplitude, onde a distorção das amplitudes devido ao espalhamento geométrico ao longo do raio de reflexão é compensado pela operaçãa de migração. Entretanto, esse processo tem um custo elevado, sendo também dependente do modelo de velocidades utilizado.

Uma rotina menos dispendiosa e mais estável em relação a erros no modelo de velocidade consiste na transformação da seção sísmica obtida com a geometria de aquisição no afastamento comum em seçōes simuladas de afastamento nulo em amplitude verdadeira. Nesse caso, as amplitudes das reflexões primárias em afastamento comum são transformadas de tal forma que o fator de espalhamento geométrico original (dependente do afastamento) é automaticamente substituído pelo correspondente fator de espalhamento geométrico do afastamento nulo. 0 coeficiente de reflexão é, no entanto, preservado, possibilitando a sua aplicação em estudos de AVO/AVA. A operação de transformação de uma seção sísmica de afastamento constante para a sua correspondente em afastamento nulo recebe o nome de migração para afastamento nulo em verdadeira amplitude (TA MZO). No caso de um meio com velocidade constante, a curva de empilhamento do MZO e a função peso se reduzem a fórmulas analíticas, minimizando o esforço computacional.

Esse trabalho possui dois objetivos fundamentais. 0 primeiro é verificar a eficácia do algoritmo de TA MZO para velocidade constante em um modelo sintético com geologia complexa baseado em um reservatório turbidífico brasileiro de idade Neo-Albiana, onde a hipótese de velocidade constante é desrespeitada. Nesse modelo, a velocidade é variável, tanto espacial quanto temporalmente. 0 modelo de velocidades utilizado para a operação de TA MZO foi encontrado através da análise de velocidade convencional realizada em intervalos regulares no domínio CMP. 0 segundo objetivo é a realização de estudos quantitativos referentes à obtenção de amplitudes resultantes da aplicação da rotina descrita acima, bem como avaliar sua utilização para a construção de gráficos de amplitude com 0 afastamento (AVO) e com o ângulo (AVA) em interfaces relevantes para a caracterização de reservatórios.

\section{MÉTODO UNIFICADO DE IMAGEAMENTO SÍSMICO EM MEIOS 2,5D}

Neste método considera-se a situaç̧ão 2,5D (BLEISTEIN, 1986) em que a propagação da onda sísmica é 3D e o modelo geológico é 2D. Seja também a subsuperfície formada pela sobreposição de camadas estratificadas, isotrópicas, e não-homogêneas, separadas por interfaces refletoras suaves, de tal forma que a propagação da onda é descrita pela teoria do raio de ordem zero para reflexões primárias num plano (BLEISTEIN, 1986). 0 exemplo mais importante é o fator de espalhamento geométrico 3D que se decompõe em duas contribuições: 0 fator de espalhamento geométrico $2 \mathrm{D}$ no plano e o fator de espalhamento da energia fora do plano.

A configuração original é a de afastamento comum composta pelos pontos médios entre fonte e receptor descritos no domínio do tempo por coordenadas ( $\xi$ ). Assume-se que a seção sísmica em afastamento comum é composta de traços analíticos $U(\xi)$, onde tvaria ao longo do tempo de registro $(0<t<\mathrm{T})$. A configuraçãa de medição de saída desta transformada é a de afastamento nulo. Ela também é composta de pontos médios nos quais fontes e geofones são coincidentes sendo descritos por coordenadas $\left(\xi_{0}, t_{0}\right)$. Na seção sísmica de afastamento nulo o traço analíico será definido como $U_{0}\left(\xi_{0}, t_{0}\right)$, lembrando que 0 traço analítico é composto do sinal sísmico registrado como parte real adicionado a sua transformada de Hilbert como parte imaginária.

0 objetivo do MZO tipo Kirchhoff é transformar as reflexões primárias encontradas na seção de afastamento comum ao longo das curvas de tempo de trânsito na sua correspondente de afastamento nulo em amplitude verdadeira. A expressão analítica que em um único passo representa essa operação é uma integral de empilhamento ponderado tipo Kirchhoff (TYGEL et al., 1998)

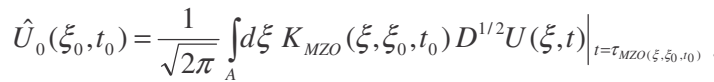

onde $t=\tau_{\text {Mzo }}\left(\xi, \xi_{\theta} t\right)$ é a curva de empilhamento para 0 MZO, $\xi$ é a coordenada do ponto médio variando ao longo da abertura $A, D^{1 / 2}$ representa 0 operador de meia derivada em tempo negativo e $K_{\text {Mod }}\left(\xi_{\text {, }} \xi_{\theta} t\right.$ d $)$ é a função peso. Conforme Schleicher et al. (1993), a 
integral (1) pode ser avaliada assintoticamente pelo método da fase estacionária (BLEISTEIN, 1984) para as altas freqüências, no ponto estacionário.

A curva de empilhamento do MZO é o lugar onde se localizam todas as contribuições na seção de afastamento comum para a determinação da amplitude em cada ponto da seção de afastamento nulo a ser simulada. Ela representa, consequentemente, a relação entre o tempo em afastamento nulo e o não nulo, podendo ser deduzida, do ponto de vista cinemático, a partir de raciocínio semelhante ao da resposta impulsiva do DMO.

Tygel e outros (1998) mostraram que a curva de empilhamento e a função peso para a migração em afastamento nulo para velocidade constante $\left(t=\tau_{M 2}\right)$ são fórmulas analíticas, sendo a primeira função do tempo no afastamento nulo $\left(\tau_{0}\right)$ e da velocidade $(V)$

$t=\frac{2 h}{v} \sqrt{1+\left(\frac{v t_{0}}{2 h}\right)^{2} a^{2}(\eta)}$,

enquanto a segunda é dada por

$K_{M Z O}\left(\xi, \xi_{0}, t_{0}\right)=\frac{a(\eta) \sqrt{t}}{h}\left(2 a^{2}(\eta)-1\right)$,

onde, em ambas as expressões acima,

$a(\eta)=\frac{1}{\sqrt{1-\frac{\eta^{2}}{h^{2}}}}, \quad|\eta|=\left|\xi-\xi_{0}\right|<h$.

Ao aplicar-se o MZO aos dados sísmicos, representa-se o refletor imageado por uma faixa migrada de largura distinta daquela original. Esse efeito é definido como distorção do pulso, ao qual relaciona-se um fator de estiramento que será denotado $m_{M Z O}$ como demonstrado em Tygel e outros (1998) o fator de estiramento no MZO é

$m_{M Z O}\left(\xi_{R}, \xi_{0 R}, t_{0 R}\right)=\cos \Theta$,

onde $\Theta$ define 0 ângulo de incidência da reflexão original no afastamento comum, ou seja o sinal de reflexão simulado em afastamento nulo é estirado em relação ao sinal sísmico original em afastamento comum ou em relação a uma seção de afastamento nulo real de um valor correspondente ao coseno do ângulo de incidência, conseqüentemente 0 conteúdo de frequência será escalonado por este valor.

Observando-se a equação para o fator de estiramento no MZO (5) percebe-se que a aplicação da migração para afastamento nulo aos dados sísmicos em afastamento comum fornece uma medida independente do ângulo de incidência $\Theta$, desde que seja possível estimar a assinatura da fonte antes e depois do MZO. Como a estimativa da assinatura da fonte é problemática, um método de obter os ângulos de reflexão para cada afastamento comum que compõem 0 experimento sísmico, mais especificamente, em estudos de AVO é executar um segundo MZO com uma funç̃ão peso modificada de forma conveniente (BLEISTEIN, 1987), TYGEL et al., 1993; BLEISTEIN et al., 1999). Este procedimento apresenta um pequeno esforço computacional, uma vez que os pesos envolvidos são multiplicadores que dependem das quantidades já calculadas pelo operador do MZO. Considerando a segunda função peso como sendo

$$
\tilde{K}_{M Z O}\left(\xi, \xi_{0}, t_{0}\right)=\cos \Theta K_{M Z O}\left(\xi, \xi_{0}, t_{0}\right),
$$

onde $K_{M Z O}\left(\xi, \xi_{0}, t\right)$ é a função peso original para 0 algoritmo do MZO, e em seguida tomando-se a razão entre as duas respostas de saída, obtidas das integrais ponderadas do MZO com os diferentes pesos $\tilde{K}_{M Z O}\left(\xi, \xi_{0}, t\right)$ e $K_{M Z O}\left(\xi, \xi_{0}, t_{0}\right)$ temse a estimativa do ângulo de incidência

$$
\frac{\tilde{U}_{0}\left(\xi_{0}, \xi_{0}, t_{0}\right)}{\hat{U}_{0}\left(\xi_{0}, \xi_{0}, t_{0}\right)}=\frac{\tilde{K}_{M Z O}\left(\xi, \xi_{0}, t_{0}\right)}{K_{M Z O}\left(\xi, \xi_{0}, t_{0}\right)}=\cos \Theta \cdot(7)
$$

\section{MODELO SINTÉTICO E A RESPOSTA SÍSMICA}

0 dado sísmico real é um 3D maríimo, com tamanho de cela de $75 \times 25 \mathrm{~m}, 306$ linhas em subsuperfície e 1.041 pontos de tiro. Uma linha sísmica migrada pós-empilhamento, na direção dip, foi selecionada a partir do dado tridimensional, englobando 650 pontos de tiro. Em seguida, uma conversão tempo versus profundidade foi realizada utilizando-se um mapa de velocidades médias para um marco sismoestratigráfico de extensão regional. Mapearam-se três refletores, com contrastes significantes de velocidade no dado em profundidade, que serviu de base para a construção do modelo da subsuperfície utilizado na geração da imagem sísmica, apresentado na Figura 1.

$\mathrm{Na}$ Figura 1 a interface plano-inclinada é o fundo do mar com profundidade variando de 100 a 500m. A segunda interface é curva representando um marco sismoestratigráfico do Oligoceno Inferior cuja profundidade varia de 2.500 a $3.700 \mathrm{~m}$. Finalmente, a última interface que aparece no modelo em profundidade de 3.000 a 4.100m é 0 topo do reservatório, estando a acumulação de óleo restrita a feição dômica segmentada por duas falhas. As velocidades intervalares, para este modelo, são $\mathrm{vl}=1.500 \mathrm{~m} / \mathrm{s}$ para 0 meio 1.; $\mathrm{v} 2=3.000 \mathrm{~m} / \mathrm{s}$, para o meio 2, que é constituído por uma intercalação de folhelhos e marga; $\mathrm{v} 3=3.400 \mathrm{~m} / \mathrm{s}$ para 0 meio 3 composto basicamente por margas; $v 4$ 


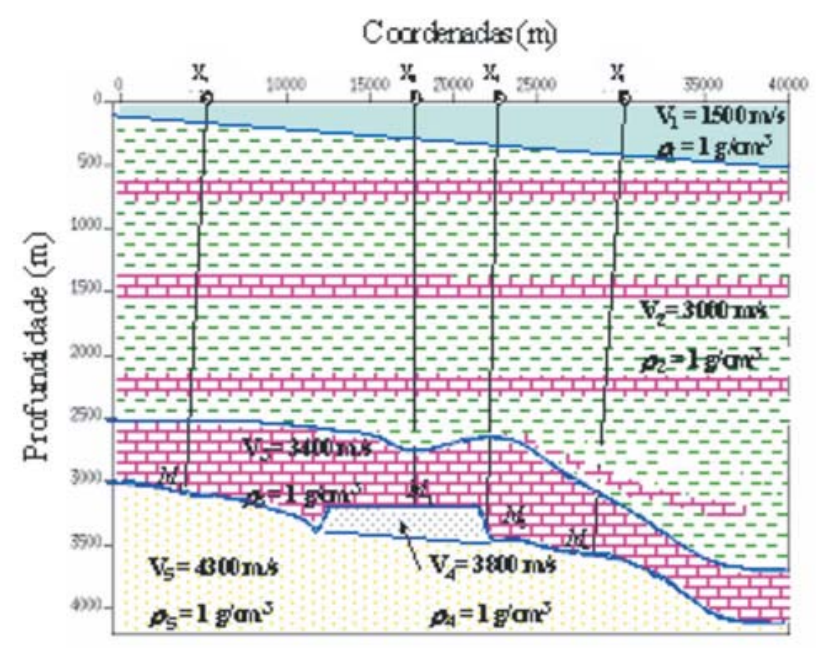

Figura 1-Modelo da Terra em profundidade Figure 1 - Earth model in depth.

$=3.800 \mathrm{~m} / \mathrm{s}$ para 0 arenito saturado com óleo; e v $5=4.300 \mathrm{~m} / \mathrm{s}$ para a areia com água. As densidades, em todos os meios, são constantes e iguais à unidade.

A imagem sísmica foi gerada no CWP/Cshot tendo sido o intervalo de tiro e de receptores de $50 \mathrm{~m}$, a banda de frequiência para construção da assinatura da fonte, de $4-72 \mathrm{~Hz}$, com o comprimento do operador de $150 \mathrm{~ms}$, a razão de amostragem de $4 \mathrm{~ms}$ e 0 tempo de registro igual a 4seg. 30 seções sísmicas sintéticas em afastamento comum foram modeladas com afastamentos variando de 100 até $3.000 \mathrm{~m}$ equiespaçados de $100 \mathrm{~m}(2 \mathrm{~h}=100,200, \ldots, 3.000 \mathrm{~m})$. Uma seção em afastamento nulo também foi imageada de modo a fornecer uma comparação com o resultado proveniente da aplicação do MZO à verdadeira amplitude.

As seç̃oes sísmicas em afastamento comum foram processadas utilizando dois fluxos de processamento (com MZO em verdadeira amplitude e com DMO FK), ou seja, as amplitudes obtidas são provenientes de dois tipos de processamento para o mesmo modelo de velocidade (Figura 2). Quatro pontos médios foram selecionados para realização das análises de AVO/AVA após a aplicação das duas sequiências de processamento mencionadas anteriormente, os $\operatorname{CMPS}_{X_{1}}$ e $X_{4}$ (Figura

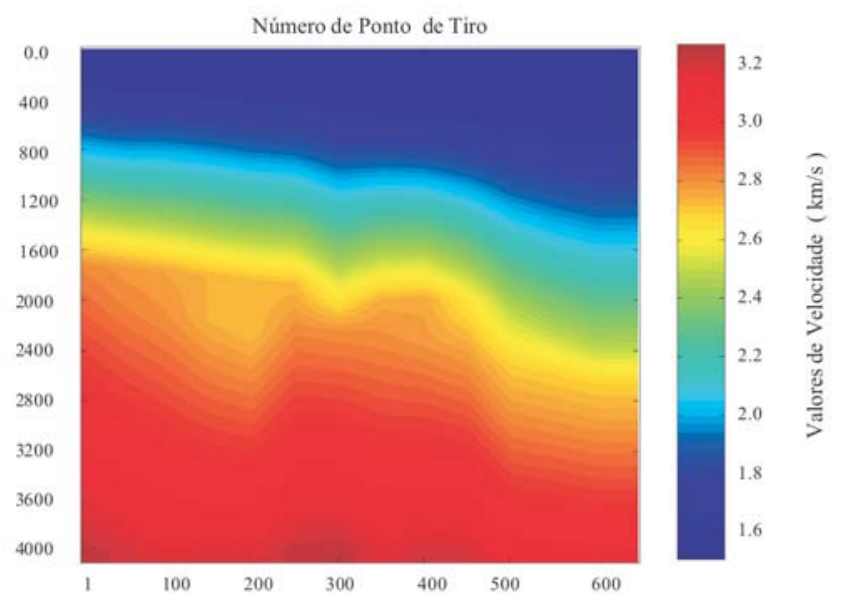

Figura 2- Mapa de Velocidades RMS.

Figure 2 - RMS velocities. 
1) estão posicionados, respectivamente, à esquerda e à direita da feição dômica, visando obter as respostas de AVO/AVA abaixo do contado óleoágua nos pontos $M_{1}$ e $M_{4}$ enquanto os CMPs $X_{2}$ e $X_{3}$ localizados sobre 0 objetivo fornecem as assinaturas de AVO/AVA nos pontos $M_{2}$ e $M_{3}$ no topo do arenito com hidrocarboneto.

Com o intuito de obter-se as respostas de AVA um segundo MZO foi executado, com peso ligeiramente diferente do primeiro, fornecendo a estimativa do ângulo de incidência, permitindo uma comparação direta entre o AVA obtido da aplicação da migração para afastamento nulo em verdadeira amplitude e o AVA teórico.

\section{DISCUSSÃO DOS RESULTADOS}

Neste estudo, como o meio de propagação é acústico, a diferença dos coeficientes de reflexão teóricos para 0 arenito saturado com água ou com óleo está exclusivamente nas velocidades dos meios envolvidos: o reservatório (arenito) e a rocha encaixante (marga). Nota-se que a resposta de AVA teórica para 0 arenito com água é aproximadamente duas vezes a resposta obtida para a areia com óleo. As comparações realizadas com a curva teórica de AVA (Figura 3) mostram que nos CMPs $X_{1}, X_{2}$ e $X_{4}$, os resultados fornecidos pelo TA MZO passam a exibir valores satisfatórios a partir do afastamento de $1.000 \mathrm{~m}$, indicando uma tendência de aumento da amplitude com 0 ângulo de incidência para as duas alternativas de processamento (TA MZO e DMO FK) tanto para água $\left(C M P s X_{1}\right.$ e $\left.X_{4}\right)$ quanto para óleo (CMP X $X_{2}$ ), o que está de acordo com os modelos de saturação, já que estes dois fluidos apresentam propriedades petrofísicas semelhantes. Apesar dos valores de amplitude resultantes do TA MZO estarem deslocados da curva teórica, no CMP X (Figura 3), eles ainda exibem um aumento da amplitude com o ângulo de incidência o que não ocorre com os valores provenientes do DMO FK.

Os gráficos de erro, comparando os resultados das duas seqüências de processamento utilizadas, estão apresentados na Figura 4, permitindo visualizar a distribuição dos erros, lembrando que esse modelo desrespeita a premissa de velocidade constante. No modelo em questão, a distância entre traços é de $50 \mathrm{~m}$. Com isso, o problema na abertura do operador torna-se crítico e os valores de amplitude passam a exibir erros muito elevados nos afastamentos curtos. No intuito de minimizar esse efeito uma interpolação de traços pode ser efetuada. Esse problema pode também estar associado à limitação na avaliação assintótica da integral de empilhamento pelo método da fase estacionária.

A comparação das respostas de AVA e dos gráficos de erro realizados entre 0 TA MZO e o DMO FK sugere que as amplitudes obtidas pela migração para afastamento nulo à verdadeira amplitude melhoram (a)

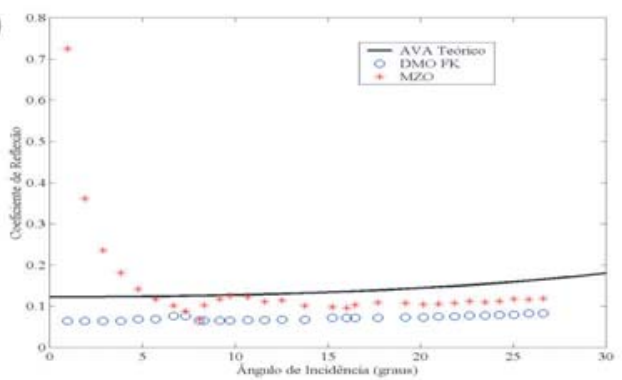

(c)

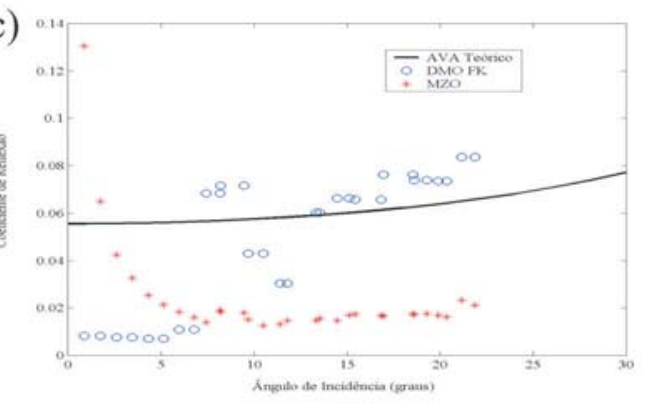

(b)

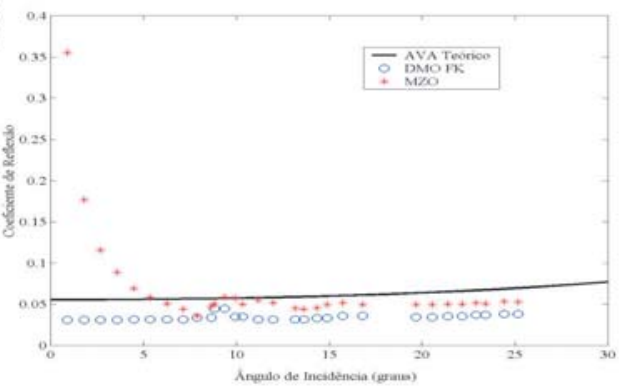

(d)

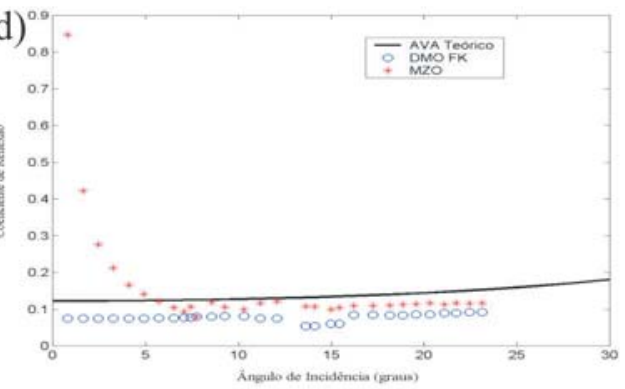

Figura 3 - Comparação entre AVA teórico e as amplitudes obtidas após TA MZO e DMO FK nos CMPs (a) X1, (b) X2, (c) X3 e (d) X4. Figure 3 - Comparison between theoretical AVA and obtained amplitudes after TA MZO and DMO FK at CMPS (a) XI, (b) X2, (c) X3 and (d) X4. 

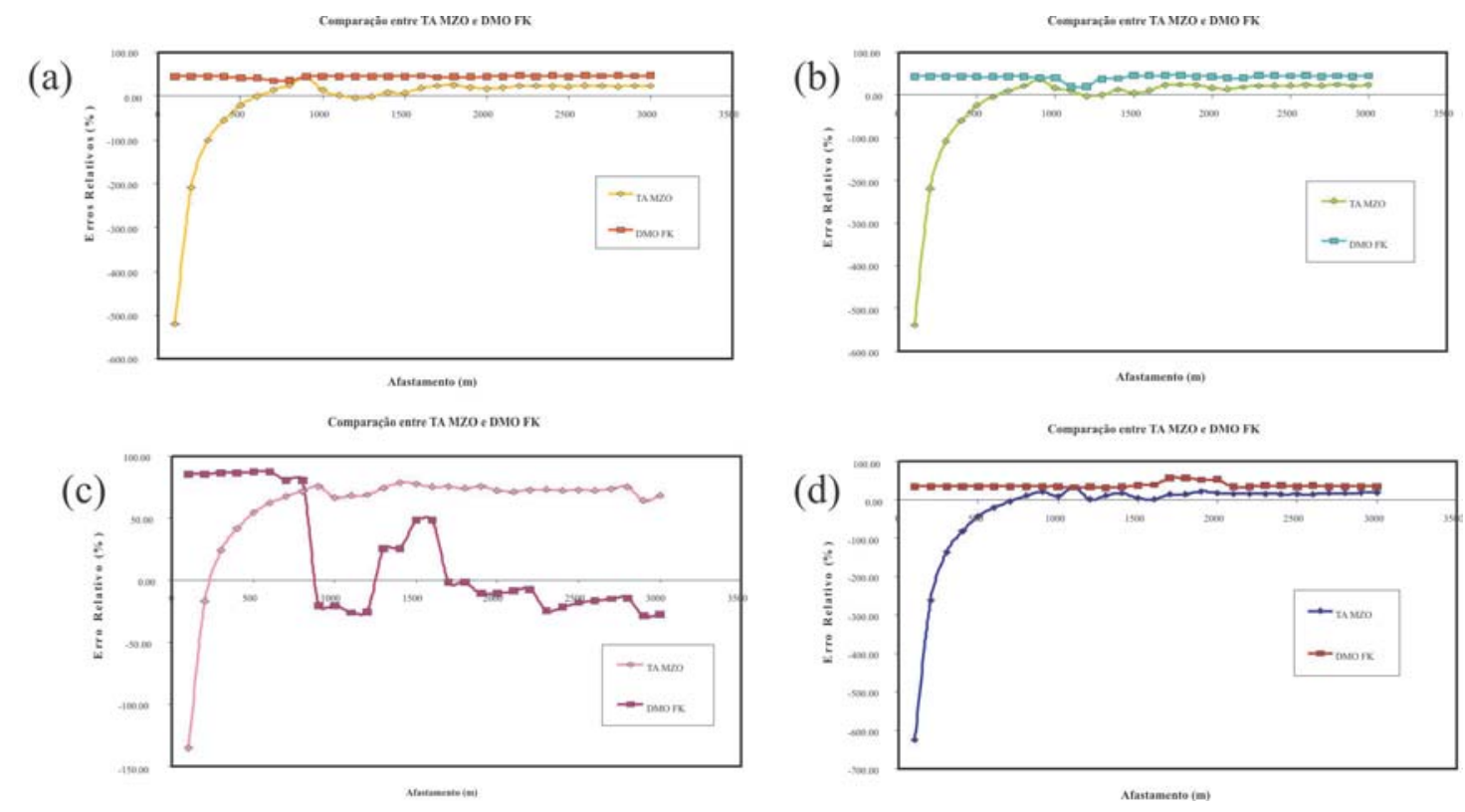

Figura 4 - Erros Relativos entre AVA teórico e as amplitudes obtidas após TA MZO e DMO FK nos CMPs (a) X1, (b) X2, (c) X3 e (d) X4. Figure 4-Relative errors between theoretical AVA and amplitudes obtained after TA MZO FK at CMPS (a) XI, (b) X2, (c) X3 and (d) X4.

a análise de AVO/AVA. Isto se deve a fato de o TAMZO fornecer melhores aproximações das perdas por espalhamento geométrico que o DMO FK.

\section{REFERÊNCIAS}

BLEISTEIN, N. Two and one half dimensional in plane wave propagation. Geophysical Prospecting, [S.I.], v. 34, p. 686-703, 1986.

BLEISTEIN, N.; COHEN, J.; JARAMILLO, H. True-amplitude transformation to zero offset of data from curved reflectors. Geophysics, Tulsa, v. 64, p. $112-129,1999$.

HUBRAL, P.; TYGEL, M.; ZIEN, H. Three-dimensional true-amplitude zerooffset migration. Geophysics, Tulsa, v. 56, p. 18-26, 1991.

; SCHLEICHER, J. A unified approach to 3D seismic reflection imaging. Part I: Basic concepts. Geophysics, Tulsa, v. 61, p. 742-758, 1996.

OLIVEIRA, A. S.; TYGEL, M.; FILPO, E. On the application of true-amplitude DMO. Journal of Seismic Exploration, Castelnau-le-Lez, v. 6, p. 279289, 1997.

; RAMOS, A. C. B. True amplitude MZO and AVO: application to real data. In: INTERNATIONAL CONGRESS OF THE
BRAZILLAN GEOPHYSICAL SOCIETY, 5., 1997, Rio de Janeiro. Proceedings. [Rio de Janeiro: SBGf], 1997. Expanded Abstract, v.1. p.223-226.

TYGEL, M. et al. Multiple Weights in diffraction stack migration. Geophysics, Tulsa, v. 59, p. 1820-1830, 1993.

TYGEL, M.; SCHLEICHER, J.; HUBRAL, P. Kirchhoff-Helmholtz theory in modelling and migration. Journal of Seismic Exploration, Castelnau-leLez, v. 3, p. 203-214, 1994 a.

P. Pulse distortion in deph migration. Geophysis, Tulsa, v. 59, p.15611569, 1994b.

Tygel, M.; Schleicher, J.; Hubral, P. Dualities involving reflectors and reflection-time surfaces. Journal of Seismic Exploration, Castelnau-leLez, v. 4, p. 123-150, 1995.

Tygel, M.; Schleicher, J.; Hubral, P. A unified approach to 3D seismic reflection imaging Part II: Theory. Geophysics, Tulsa, v. 61, p. 759-775, 1996.

Tygel, M.; Schleicher, J.; Hubral, P. 2,5D Kirchhoff MZO in laterally inhomogeneous media. Geophysics, Tulsa, v. 63, p.557-573, 1998. Tygel, M. et al. Kirchhoff imaging for AVO/AVA. The Leading Edge, [S.I.], v. 18, p. 940-945, 1999. 


\section{NOTAS SOBRE OS AUTORES}

Angela Cristina Romero Vasquez é formada em Desenho Técnico (CSA-RJ/1980), graduada em Geologia (UFRJ/1986), e Mestre em Ciência e Engenharia do Petróleo (UNICAMP/1999). Empregada da PETROBRAS desde 1987 onde participou da aquisição de dados sísmicos na Bahia, no desenvolvimento de novas tecnologias, no processamento sísmico convencional e especial e atualmente trabalha no gerenciamento de projetos de exploração. É geofísica da Unidade de Negócios de Exploração no Ativo de Campos e Espírito Santo, onde colabora no planejamento e acompanhamento de projetos de exploração como representante técnica da PETROBRAS em parcerias.

Adelson Santos de Oliveira é bacharel e mestre em física nuclear pela Universidade Federal Fluminense e doutor em geofísica (sísmica) pela Universidade Federal da Bahia (1990). Desde 1987 trabalha na Petrobras, estando lotado no Setor de Estudos Geofísicos desde 1990. Já preparou e ministrou dentro da Petrobras os cursos DMO Teoria e Prática e Filtragem Multicanal, também ajudou a preparar e ministrar o curso Processamento com Consistência em Superfície. Seus principais interesses são inversão sísmica, ałualmente com enfoque em eliminação de ruído, condicionamento de dados e imageamento.

Martin Tygel é formado em Física (UERJ/1969), Mestre em Matemática (PUC-Rio/1973) e PhD em Matemática (Stanford University/ 1979). É Professor da UNICAMP desde 1984, tendo lecionado na UFRN (Natal/1979-1980), PPPG-UFBa (Salvador/1981-1983) e Instituto de Geofísica da Universidade de Karlsruhe (Alemanha) em 1990. É detentor dos prêmios Schlumberger (EAGE/2002) e Zeferino Vaz (UNICAMP/1997 e 2003). É fundador do Laboratório de Geofísica Computacional da UNICAMP. 0 Laboratório é um dos membros do Consórcio Wave Inversion Technology (WIT), com sede em Karlsruhe. É consultor da Petrobras desde 1991 em processamento e imageamento sísmicos. É Editor Associado da Revista Geophysics (SEG).

Lúcio T. Santos é graduado em Matemática Aplicada (UNICAMP/1982), Mestre em Matemática Aplicada (UNICAMP/1985), Doutor em Engenharia Elétrica (UNICAMP/1991) e Livre-Docente em Otimização (UNICAMP/1999). De 1985 a 1988 foi Professor Assistente na USP/São Carlos e desde 1998 é professor no Departamento de Matemática Aplicada do IMECC/UNICAMP, atuando nos grupos de Geofísica Computacional e Otimização. De Agosto/1994 a Setembro/1995 fez Pós-Doutorado na Rice University (EUA) e durante Janeiro/Fevereiro de 1998, 1999 e 2001 foi Professor Visitante no Instituto de Geofísica da Universidade de Karslruhe (Alemanha). Seus interesses profissionais incluem modelamento e imageamento sísmicos e otimização não-linear. Suas pesquisas recentes envolvem novas aproximações para 0 coeficiente de reflexão P-P, atributos alternativos para a análise de AV0, expressões multiparamétricas para tempos de trânsito e métodos de diferenças finitas para a equação Iconal. É membro da SBGf e da SEG. 